\title{
Avaliação do autoconhecimento dos fatores de risco cardiovascular em população da periferia da cidade de Vassouras: dados preliminares
}

Evaluation of self-knowledge of cardiovascular risk factors in the population of the outskirts of the city of Vassouras: preliminary data

Caio Teixeira dos Santos ${ }^{\dagger *}$, Raul Ferreira de Souza Machado ${ }^{\dagger}$, Thais Lemos de Souza Macêdo ${ }^{\dagger}$, Dandhara Martins Rebello ${ }^{\dagger}$ João Paulo Brum Paes ${ }^{\dagger}$ Ivana Picone Borges de Aragão

Como citar esse artigo. dos Santos, C.T.; Machado, R.F.S.; Macêdo, T.L.S.; Rebello, D.M.; Paes, J.P.B.; de Aragão, I.P.B.A. Avaliação do autoconhecimento dos fatores de risco cardiovascular em população da periferia da cidade de Vassouras: dados preliminares. Revista de Saúde. 2019 Jul./Dez; 10 (2): 12-17.

\section{Resumo}

As doenças cardiovasculares (DCV) se enquadram entre as principais causas de morte no mundo ocidental, embora sua incidência possa ser reduzida por meio da prevenção primária e da redução a exposição a fatores de risco cardiovasculares (FRCV). Este estudo verificou o autoconhecimento sobre FRCV em moradores de periferia da cidade de Vassouras, no Estado do Rio de Janeiro. Trata-se de um estudo observacional e transversal, cujos dados foram coletados entre 2017 e 2018, por meio de questionário estruturado, contendo 32 perguntas de respostas rápidas, em indivíduos com idade $\geq 20$ anos. A amostra foi constituíd por 150 indivíduos, com média de idade de 46,6 anos, sendo 96 mulheres e 54 homens. O valor médio do colesterol foi de 192mg/dl. FRCV informados: 28 indivíduos fumantes, 35 ex-fumantes, 65 hipertensos, 25 portadores de hiperglicemia, sendo com 24 deles fazendo uso de medicação. Infarto agudo do miocárdio (IAM) prévio foi informado por 18 indivíduos, IAM em pais/irmãos por 27 e nas mães/irmãs, por 31 indivícuos. O indice de massa corporal (IMC) médio foi 27,60. Da amostra, 93 negaram regularidade de exercícios físicos, 86 afirmaram cansaço, 58 palpitação, 52 dispneia, 26 desmaio sem explicação, 75 dores nas pernas ao caminhar, 31 dores no peito em esforço e 21 em repouso. Registrados 24 casos de hipertensão gestacional, 7 de pré-eclâmpsia, 37 na menopausa, 18 histerectomizadas, 14 ooferectomizadas, 2 em reposição hormonal e 110 negaram consulta regular com cardiologista. Autopercepção do estresse foi relatado por 47 indivíduos, que informaram ser muito frequente, enquanto para 15 foi pouco frequente. A população estudada demonstrou significativo autodesconhecimento em relação aos FRCV, o que pode impactarno desenvolvimento DCV. Constatou-se assim, a importancia da prevenção primária para as DCV.

Palavras-chave: Doenças Cardiovasculares, Prevenção Primária, Fatores de Risco.

\begin{abstract}
Cardiovascular diseases (CVD) are among the leading causes of death in the Western world, although their incidence can be reduced through primary prevention and reduced exposure to cardiovascular risk factors (CVRF). This study aimed to verify the self-knowledge of CVRF in residents of the outskirts of Vassouras, Rio de Janeiro. Observational and crosssectional study, whose data were collected between 2017 and 2018, through an anonymous structured questionnaire, containing 32 quick answer questions, in individuals aged $\geq 20$ years. We evaluated a total of 150 individuals with a mean age of 46.6 years, 96 women and 54 men. Average cholesterol value of 192mg / dl. Reported CVRF: 28 smokers, 35 former smokers, 65 hypertensive, 25 with hyperglycemia and 24 on medication. Previous acute myocardial infarction (AMI) was reported by 18 subjects, AMI 27 in parents / siblings and 31 in mothers / sisters, the mean body mass index (BMI) was 27.60. Of the sample, 93 denied regular exercise, 86 reported tiredness, 58 palpitation, 52 dyspnea, 26 unexplained fainting, 75 pains in the legs while walking, 31 chest pains in stress and 21 at rest. There were 24 cases of gestational hypertension, 7 of preeclampsia, 37 at menopause, 18 hysterectomized, 14 ovariectomized, 2 hormone replacement and 110 denied regular consultation with a cardiologist. Self-perception of stress: 47 individuals reported being very frequent, 15 uncommon, 45 sometimes, 17 almost never, 23 did not feel and 2 could not answer. The population studied showed high self-knowledge regarding CVRF, which impacts on exposure to CVD development, with the importance of primary prevention for CVD to be worked in the community.
\end{abstract}

Keywords: Cardiovascular Diseases, Primary Prevention, Risk Factors 


\section{Introdução}

Doenças Cardiovasculares (DCV) estão entre as principais causas de morte em todo o mundo e no Brasil. Nas últimas décadas, segundo dados da Organização Mundial de Saúde (OMS) e do DATASUS ${ }^{1}$, cerca de $30 \%$ dos óbitos, para todas as faixas etárias, são resultados dessa patologia - das 50 milhões de mortes, as DCV representam 17 milhões $^{2}$. Em países em desenvolvimento como o Brasil, a DCV tende a aumentar devido ao processo de urbanização, que consequentemente ocasiona um aumento da incidência de tabagismo, obesidade, hipertensão e dislipidemias. Outro ponto a ser considerado é a diminuição da mortalidade por causas infecto-parasitárias, o que aumentou a expectativa de vida ${ }^{3}$.

Acerca da incidência das DCV, grande parte das situações podem ser evitadas se houver prevenção primaria e secundária ${ }^{4}$. A falta de informação sobre o assunto representa um ponto fundamental na não diminuição da incidência, visto que a identificação precoce, o tratamento adequado e o monitoramento dos FRCV permitem a redução da morbimortalidade, com consequente diminuição de custos públicos ou privados. Há consenso de que o manejo adequado dos FRCV pode reduzir os eventos cardiovasculares ${ }^{5}$. Nos países desenvolvidos, foi analisada uma proporcionalidade indireta entre os níveis socioeconômicos e a incidência e prevalência de cenários de DCV. Entretanto, macrorregiões no Brasil, por exemplo, tendem a ter uma prevalência maior, muito provavelmente, devido à falta de conhecimento sobre DCV por parte da população ${ }^{6}$.

$\mathrm{O}$ rastreamento do $\mathrm{RCV}$ tem como intuito, de acordo com Kones ${ }^{7}$, atrasar ou limitar o primeiro evento em indivíduos, que consiste na prevenção primária, antes do diagnóstico de doença cardíaca.

O objetivo do presente estudo foi avaliar o autoconecimento dos fatores de risco para as doenças cardiovasculares numa população de periferia da cidade de Vassouras no Estado do Rio de Janeiro.

\section{Material e Métodos}

Estudo observacional e transversal ocorrido entre março de 2017 e novembro de 2018. Os critérios de inclusão para o estudo foram o participante residir nas localidades Itakamosi e de Ipiranga, na perifieria da cidade de Vassouras; ter idade $\geq 20$ anos de idade, estabelecida a partir dos dados obtidos no Departamento de Informática do Sistema Único de Saúde (DATASUS).

O estudo foi aprovado pelo Comitê de Ética da Universidade de Vassouras sob parecer do CEP número 1.883. 015, de 28 de dezembro de 2016.

A escolha das localidades cujos moradores iriam participar da pesquisa, teve como justificativa a facilidade de acesso dos alunos da iniciação científica aos locais, uma vez que a Universidade de Vassouras disponibiliza o transporte para os alunos do primeiro, segundo e terceiro período do curso de medicina participantes de um projeto que assiste moradores das citadas localidades. O outro aspecto foi os participantes terem piores condições socioeconomicamente, que de acordo com dados da literatura, indicam uma prevalência e incidência maior de FR e de eventos $\mathrm{CV}$, que podem ser prevenidos primariamente ou diagnosticados precocemente a partir de análise dos FR e do conhecimento dos mesmos.

O número total de moradores foi obtido através do número de indivíduos registrados e visitados pelos alunos de primeiro ao terceiros período do curso de medicina, no projeto desenvolvido pela Universidade de Vassouras.

Os dados foram coletados por meio de questionário anônimo, contendo respostas rápidas, de multipla escolha, estruturado, com o objetivo de preservar a identidade dos participantes. Os alunos de iniciação científica foram os responsáveis em aplicar os questionários aos moradores de ambos as localidades.

O questionário continha 45 perguntas em relação a dois pontos: 1) o autoconhecimento dos fatores de risco cardiovascular, incluindo: idade; tabagismo (sim ou não); hipertensão (sim ou não, desconhecimento); dislipidemia e valor do colesterol total, LDL e HDL (sim, não, desconhecimento); história familiar de doença arterial coronariana ou acidente vascular cerebral em parentes de primeiro grau (sim, não, desconhecimento); diabetes e valor da glicemia (sim, não, desconhecimento); valor do índice de massa corporal (IMC), peso e altura (valor, desconhecimento); prática de exercícios físicos maior que 150 minutos por semana (sim, não); histórico de infarto ou angina pectoris (sim, não, desconhecimento); sintomas de fadiga ou palpitações ou falta de ar ou desmaio ou dor nas pernas ao caminhar (sim, não); sintomas de desconforto no peito, pescoço, mandíbulas ou ombro com esforço ou repouso (sim, não), sendo considerado aquelas com 2 ou mais sintomas; gravidez ( $\operatorname{sim}$, não); hipertensão na gravidez (sim, não, desconhecimento, não aplica), pré-eclampsia (sim, não, desconhecimento, não aplica); diabetes na gravidez ( sim, não, desconhecimento, não aplica); menopausa (sim, não, desconhecimento); útero removido (sim, não); ovários removidos (sim, não); reposição hormonal (sim, não, não aplica); prevenção ginecológica regularmente (sim, não), prevenção cardiológica regularmente (sim, não); auto percepção do estresse (nunca, raramente, pouco frequente, frequentemente, muito frequente), última pergunta, essa, sendo relevante para a análise da ocorrência de eventos cardiovasculares, ressaltado por Loures et al. (2002) - o aumento significativo da frequência de eventos está associada ao estresse 
mental.

2) as condições socioeconômicas dos participantes, incluindo: ser beneficiário de algum programa social (como bolsa família, benefício de assistência, etc.); residência em zona urbana ou rural; moradia própria, alugada ou cedida; com acabamento, sem acabamento, palafita ou pau a pique; presença de rede de esgoto, fossa, banheiro (quantos), chuveiro e luz; sobre o abastecimento de água (por encanamento, de mina e poço) e coleta de lixo (mais de uma vez por semana, semanal, quinzenal, mensal ou não presença de coleta); número de cômodos; grau de escolaridade (não alfabetizado, ensino fundamental incompleto, ensino fundamental completo, ensino médio incompleto, ensino médio completo, ensino superior incompleto e ensino superior completo); se o pesquisado trabalha ou se é estudante; quantas pessoas moram na mesma residência; e sobre a renda mensal o indivíduo (menos de $\mathrm{R} \$ 400,00$, entre $\mathrm{R} \$ 400,00$ e $\mathrm{R} \$ 880,00$, entre $\mathrm{R} \$ 880,00$ e $\mathrm{R} \$ 1700,00$, entre $\mathrm{R} \$ 1700,00$ e $\mathrm{R} \$ 2000,00$, entre $R \$ 2000,00$ e $R \$ 2300,00$ ) e quantas pessoas vivem com a renda.

As variáveis analisadas correspondem a cada resposta das perguntas contidas no questionário, que foram inseridas em um banco de dados no programa Excel. As variáveis categóricas foram analisadas por porcentagens com Figuras e e tabelas, e médias para as variáveis contínuas. Cada questionário foi analisado separadamente para obter-se uma análise descritiva dos dados.

\section{Resultados}

Participaram da pesquisa 150 moradores. A média de idade foi 46,6 anos, sendo 96(64\%) mulheres e $54(36 \%)$ homens (Figura 1). Tabagismo foi mencionado por $28(18,66 \%)$ moradores, sendo $35(23,33 \%)$ extabagistas (Figura 2).

De acordo com o autoconhecimento acerca dos fatores de risco cardiovascular, foram obtidas as seguintes informações: 65 (43,33\%) indivíduos referiram pressão arterial >120/80 mmHg; $113(75,33 \%)$ afirmaram já terem realizado dosagem de colesterolemia total, sendo que desses, $25(16,66 \%)$ afirmaram valores $>200 \mathrm{mg} /$ dL; o valor médio do colesterol total informado na população estudada foi de $192 \mathrm{mg} / \mathrm{dL} ; 115$ (76,66\%) não souberam informar o valor do colesterol HDL e

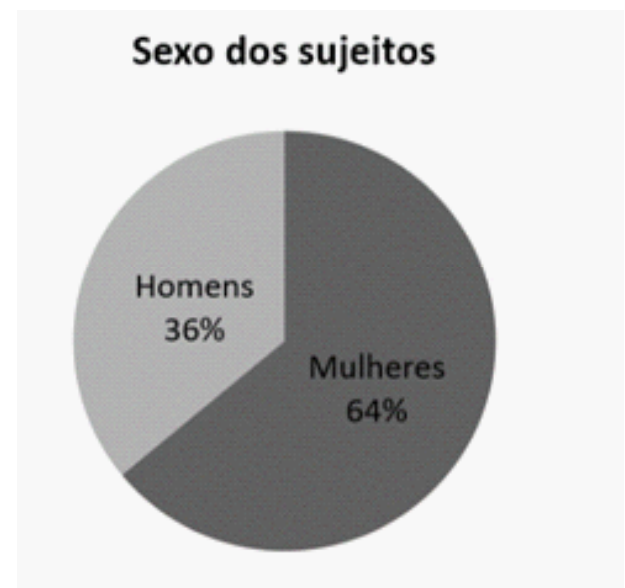

Figura 1. Sexo dos participantes da amostra

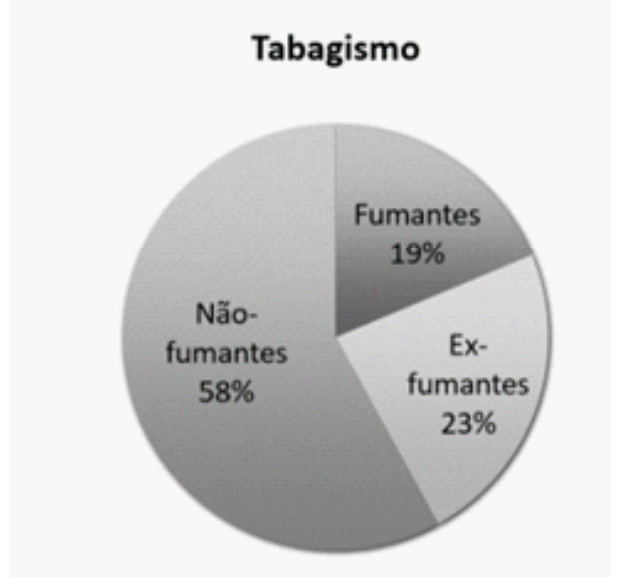

Figura 2. Tabagismo nos participantes

\section{Presença de Fatores de Risco}

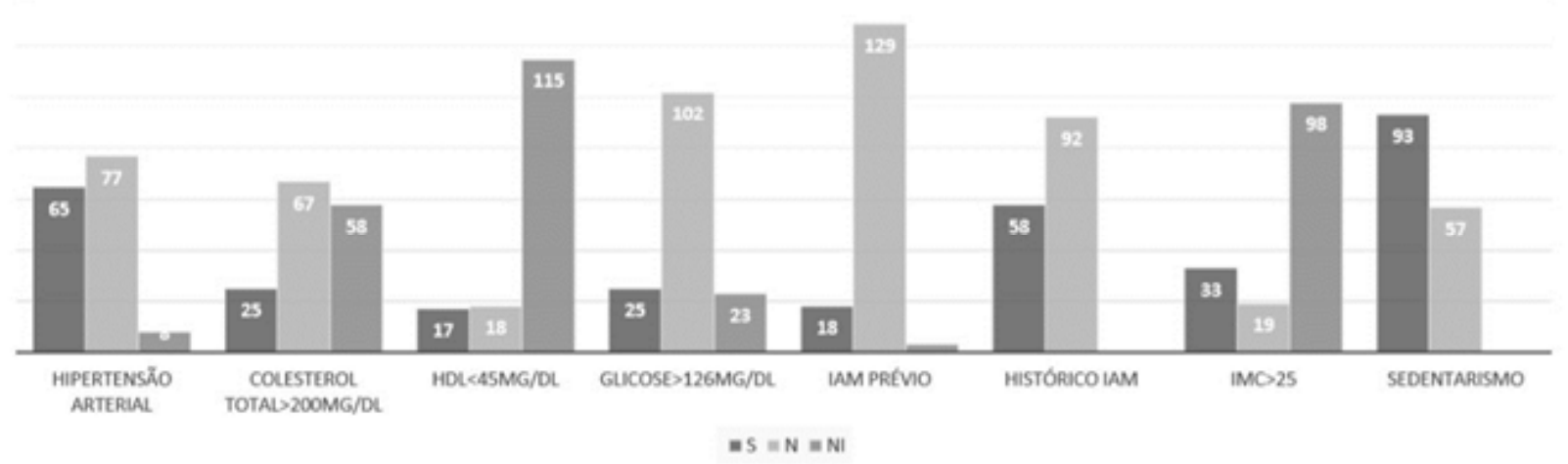

Figura 3. Esquematização da presença de fatores de risco no grupo estudado. 


\section{Percepção de Sinais e Sintomas agravantes}

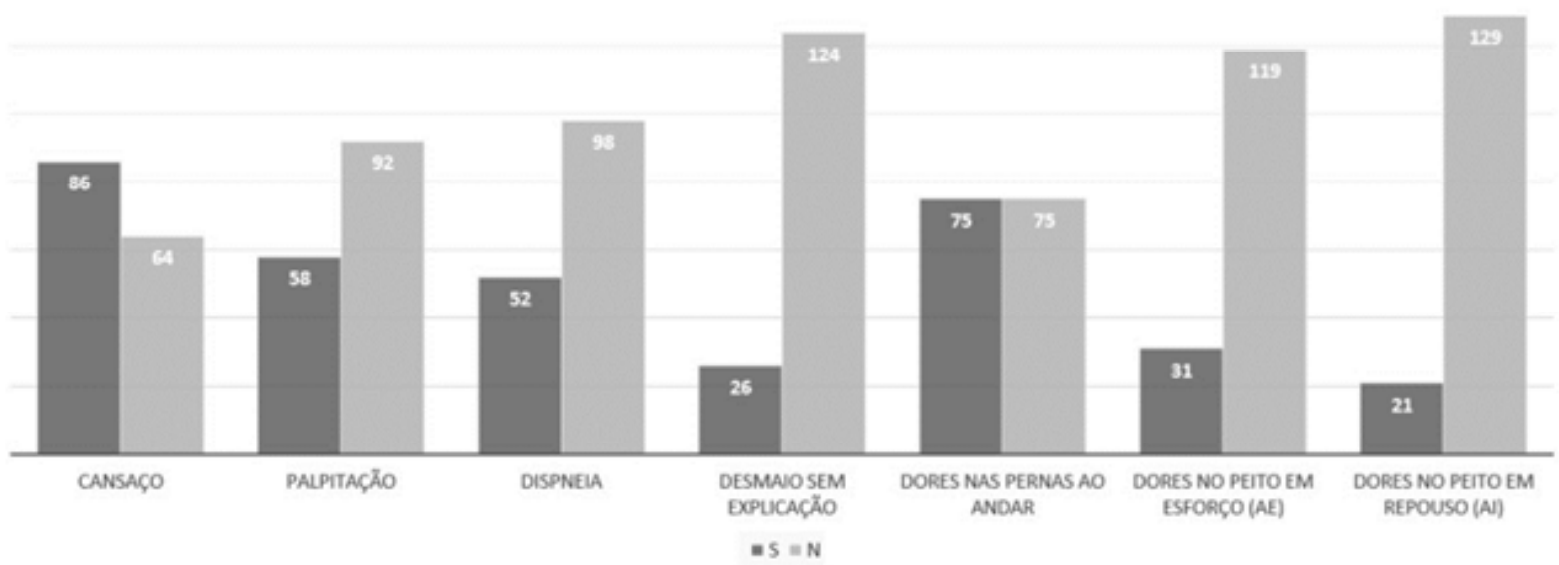

Figura 4. Esquematização da auto percepção de sinais e sintomas da amostra.

\section{Dados femininos}

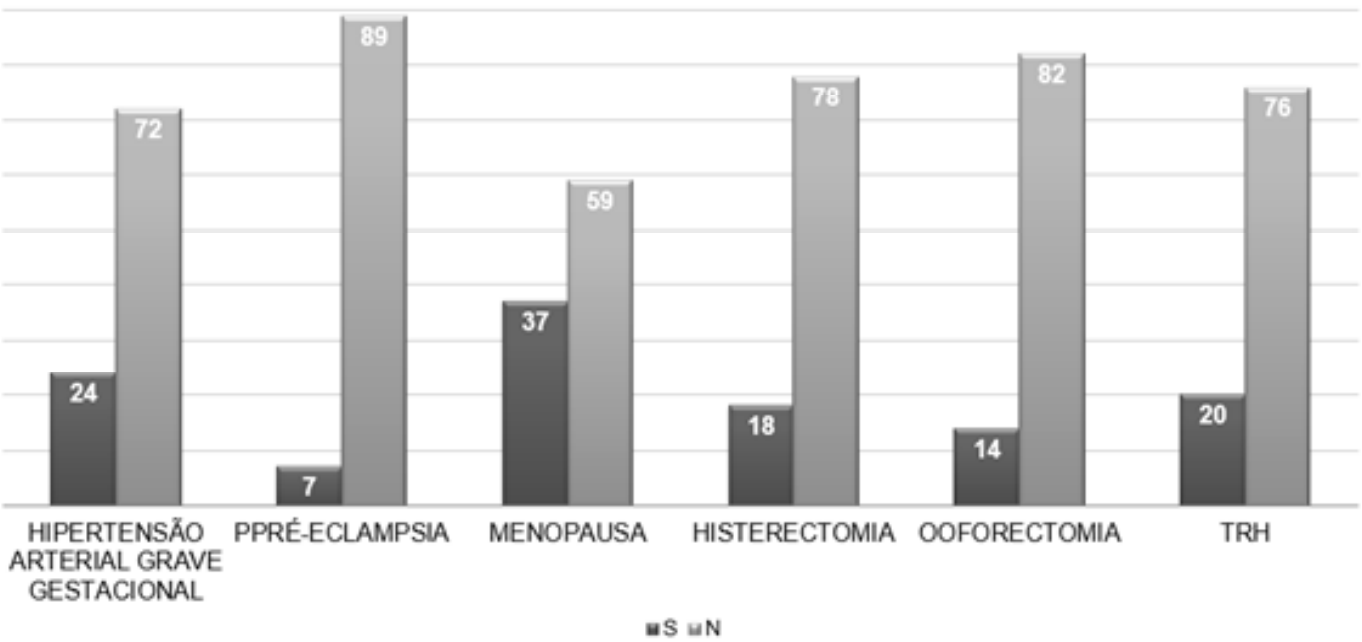

Figura 5. Distribuição dos dados clínicos da população feminina.

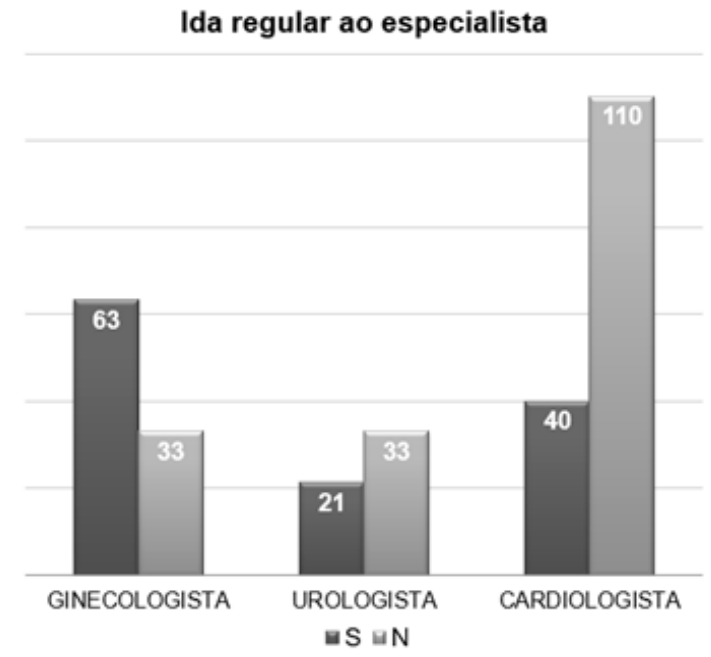

Figura 6. Distribuição dos aspectos de acompanhamento clínico da amostra. 


\section{Auto-percepção do estresse}

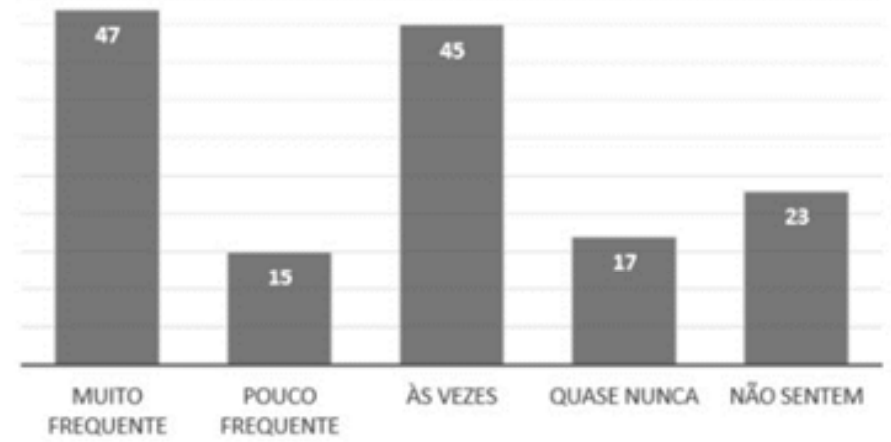

Figura 7. Esquematização da auto percepção do estresse da amostra estudada.

$17(11,33 \%)$ afirmaram HDL $<45 \mathrm{mg} / \mathrm{dL} ; 102(68 \%)$ negaram glicemia $>126 \mathrm{mg} / \mathrm{dL} ; 129(86 \%)$ participantes negaram IAM prévio com 18 (12\%) afirmativas; 27 (18\%) afirmaram história familiar de IAM em pais ou irmãos e 31 (20,66\%), em mães ou irmãs; $65 \%$ dos indivíduos desconheciam se IMC $>25$ (sobrepeso) e $19(12,66 \%)$ afirmaram valores normais; a média do IMC calculado foi de 27,60 , porém $40(26,66 \%)$ não souberam informar altura; sedentarismo informado por $93(62 \%)$ (Figura 3$)$.

Os sintomas a seguir foram relatados pelos moradores avaliados: $86(57,33 \%)$ relatos de cansaço; $58(38,66 \%)$ de palpitação; $52(34,66 \%)$ dispneia; 26 $(17,33 \%)$ desmaio sem explicação; $75(50 \%)$ dores nas pernas ao caminhar, $31(20,66 \%)$ dor torácica em esforço e 21 (14\%) em repouso (Figura 4). Total de 24 (25\%) mulheres informaram HAS gestacional com 07 (7,29\%) pré-eclâmpsia; 37 (38,54\%) em menopausa; 18 $(18,75 \%)$ com passado de histerectomia e $14(14,5 \%)$ de ooforectomia; $76(79,16 \%)$ negaram terapia de reposição hormonal (TRH) (Figura 5).

Questionados quanto ao comparecimento em consultas médicas com especialistas: $40(26,67 \%$ do total) afirmaram ir ao cardiologista; $63(67,7 \%$ das mulheres) ao ginecologista e 21 (37,5\% dos homens) ao urologista. As respostas "não" e "não aplicável" foram agrupadas em "não" (Figura 6). Em relação à autopercepção do estresse, observou-se que 47 $(31,33 \%)$ afirmaram ser muito frequente; $15(10 \%)$ pouco frequente; 45 (30\%) às vezes; $17(11,33 \%)$ quase nunca; 23 (15,33\%) negaram (Figura 7).

\section{Discussão}

A população das cidades vem crescendo com o passar dos anos, e com isso aumentam também os índices de DCV nessas localidades em decorrência do estilo de vida. Além disso, em grande parte pode se observar a necessidade de desenvolvimento de um sistema de prevenção primária e secundária, o que facilitaria a redução da incidência das DCV e a sobrecargados serviços especializados. Muitas vezes, os casos levados ao especialista poderiam ter sidos resolvidos na atenção básica. $\mathrm{O}$ rastreamento em medicina, em particular nas doenças cardiovasculares, tema de estudo, tem sua importancia em garantir a identificação do perfil de uma população e o encaminhamento para os serviços adequados, responsáveis pela prevenção primária, secundária e terceária. Permite também a criação de programas de conscientização, possibilitando ao indivíduo conhecer os FR, despertando o automonitoramento e a multiplicação do conhecimento.

A Organização Mundial de Saúde (OMS) vem propondo uma abordagem que busca correlacionar a prevenção e o controle dos FRCV, pois muitas vezes os indivíduos acabam compartilhando mais de um FR, como a HAS, tabagismo, hipercolesterolemia, sedentarismo, dieta inadequada e a obesidade, o que gera ainda maiores chances para a ocorrência de evento agudo ou desenvolvimento de doença crônica grave.

Outros estudos mostram ainda que as doenças cardíacas possuem alto custo financeiro no Brasil já há alguns anos, como por exemplo, no de 2015 onde foram gastos em média $\mathrm{R} \$ 56,2$ bilhões em casos de insuficiência cardíaca, IAM, fibrilação atrial e HAS no país ${ }^{8}$.

As DCV são também muitas vezes limitantes além de custosas ao sistemasde saúde, e afetam o bemestar e a produtividade dos indivíduos, tendo fortes relações com outras comorbidades, como a obesidade por exemplo ${ }^{8}$. 
Ao se tratar de regiões de periferia, pode-se notar menores índices de escolaridade e renda, que segundo estudos previamente realizados, quando atrelados a outros determinantes sociais de saúde como gênero, etnia, trabalho e estilo de vida da população influenciam diretamente a presença e distribuição de FR e também a patogênese das DCV. Além disso, a literatura atual também evidencia um aumento da mortalidade por afecções cardíacas entre pessoas com menor nível socioeconômico?.

Em relação a educação, saúde e saneamento básico a influência nos FRCV se mostra presente na redução desses determinantes na periferia, o que torna a prevalência dessas doenças ainda maior nessas regiões. Estudos apontam que em relação a educação, quanto maior é o nível de escolaridade menor é o acometimento pelas DCV e seus $\mathrm{FR}^{10}$, assim como menor a prevalência de diabetes e sedentarismo, que são fatores muitas vezes associados a problemas cardíacos ${ }^{11}$.

\section{Conclusões}

Pode-se observar a grande presença dos fatores de risco para doenças cardiovasculares na população estudada. Além disso, baseando-se nos resultados, é visto o pouco conhecimento acerca do assunto. Uma parcela significativa do grupo se apresenta com doenças em níveis não controlados, e cursam com sintomas, sendo assim, é necessário o apoio com equipes multidisciplinares na região, para que seja feita a conscientização sobre os riscos, o acompanhamento, e o tratamento das doenças, visando reduzir a morbidade e mortalidade na área em questão.

\section{Referências}

1. Ministério da Saúde. Plano de ações estratégicas para o enfrentamento das doenças crônicas não transmissíveis (DCNT) no Brasil, 2011-2022. Brasília: Ministério da Saúde; 2011. [Acesso em 2012 jun 17]. Disponível em: <http:// portal.saude.gov.br/portal/saude/profissional/area.cfm?id area=1818.

2. Lima JCC. As dislipidemias e suas avaliações laboratoriais. Hiperativa [revista em Internet] 2014 abril-junho. [acesso em 10 de novembro de 2016]. Disponível em: http://departamentos.cardiol.br/dha/revista/6-2/006.pdf.

3. Correia BR, Cavalcante E, Santos, E. A prevalência de fatores de risco para doenças cardiovasculares em estudantes universitários. Revista da Sociedade Brasileira de Clinica Médica. [revista em Internet] 2015 janeiro-fevereiro. [acesso em 10 de novembro de 2016.] Disponível em: http://www.sbcm.org. $\mathrm{br} /$ revistas/RBCM/RBCM-2010-01.pdf\#page $=26$.

4. Godoy MF, Lucena JM, Miquelin AR, Paiva FF, Oliveira LQ, Augustin JJL. Mortalidade por doenças cardiovasculares e níveis socioeconômicos na população de São José do Rio Preto, estado de São Paulo, Brasil. Arq. Bras. Cardiol. [revista em Internet] 2017 fevereiro.[acesso em em 13 Nov. 2016. http://dx.doi.org/10.1590/S0066-782X2007000200011.] Disponível em $\quad<$ http://www.scielo.br/scielo.php?script=sci arttext\&pid=S0066782X2007000200011\&lng=en\&nrm=iso>

5. Ishitani LH, Francoll GC, Perpétuo IHO, França E. Desigualdade social e mortalidade precoce por doenças cardiovasculares no Brasil. Rev. Saúde. [revista em Internet] 2006 agosto. [Acesso em 13 Nov. 2016. http://dx.doi.org/10.1590/S0034-89102006000500019.]. Disponível em $\quad<$ http://www.scielo.br/scielo.php?script=sci_arttext\&pid=S0034-
$89102006000500019 \& \operatorname{lng}=\mathrm{en} \& \mathrm{nrm}=\mathrm{iso}>$.

6. Simão AF, Precoma DB, Andrade JP. I Diretriz de Prevenção Cardiovascular Arq Bras Cardiol. [revista em Internet] 2013 dezembro. [Acesso em 13 Nov. 2016]. Disponível em <http://www.scielo.br/scielo.php?pid=S0066$782 \mathrm{X} 2013004500001 \&$ script $=$ sci arttext $>$.

7. Kones R. Is prevention a fantasy, or the future of medicine? A panoramic view of recent data, status and direction in cardiovascular prevention. Ther Adv Cardiovasc Dis. [revista em Internet] 2011fevereiro. [acesso em 19 de maio de 2019]. Disponívelem<https://www.ncbi.nlm.nih.gov/ pubmed/21183531>

8. Stevens B, Pezzullo L, Verdian L, Tomlinson J, George A, Bacal F. Os Custos das Doenças Cardíacas no Brasil. Arq. Bras. Cardiol. [revista em Internet] 2018 Julho.[acesso em 17 de maio de 2019]. Disponível em $<$ http://www.scielo.br/scielo.php?script=sci arttext\&pid=S0066$782 \mathrm{X} 2018001300029 \& \operatorname{lng}=\mathrm{en} \& \mathrm{nrm}=\mathrm{iso}>$.

9. Medeiros CRG, Meneghel SN, Gerhardt TE. Desigualdades na mortalidade por doenças cardiovasculares em pequenos municípios. Ciência e Saúde Coletiva. [revista em Internet]2012. [acessado em 19 de maio de 2019]. Disponível em < https://doi.org/10.1590/S1413-81232012001100012>

10. Santos HC, Nascimento RM, Mill JG, Krieger JE, Pereira AC.Selfdeclared ethnicity associated with risk factors of cardiovascular diseases in an urban sample of the Brazilian population: The role of educational status in the association. Int J Cardiol. [revistaem Internet] 2013 abril. [acessoem 19 de maio de 2019] Disponível em <https://doi.org/10.1016/j. ijcard.2013.04.115>

11. Costa RP, Ferreira PAM, Junior FCM, Ferreira ASP, Ribeiro VS Nascimento GC.Fatores de Risco Cardiovascular e sua Relação com o Nível de Escolaridade numa População Universitária. Int J Cardiovasc Sci. [revista em Internet] 2015 maio-junho. [acesso em 19 de maio de 2019]. Disponível $\mathrm{em}<\mathrm{http}$ //bases.bireme.br/cgi-bin/wxislind.exe/iah/online/?IsisScript=iah/ iah. $x$ is \&src $=$ google \&base $=$ LILACS\&lang $=$ p\&nextAction $=1$ nk\&exprSearch $=775246 \&$ indexSearch $=$ ID 